Connecting directional limb movements to vowel fronting and backing

\author{
Vainio, Lari
}

2019-10-15

Vainio , L , Tiainen , M , Tiippana , K \& Vainio , M 2019 , ' Connecting directional limb movements to vowel fronting and backing ' , Neuroscience Letters , vol. 711 , 134457 . https://doi.org/10.1016/j.neul

http://hdl.handle.net/10138/318720

https://doi.org/10.1016/j.neulet.2019.134457

cc_by_nc_nd

acceptedVersion

Downloaded from Helda, University of Helsinki institutional repository.

This is an electronic reprint of the original article.

This reprint may differ from the original in pagination and typographic detail.

Please cite the original version. 


\title{
Connecting directional limb movements to vowel fronting and backing
}

\author{
Vainio, L., Tiainen, M., Tiippana, K., \& Vainio, M
}

Lari Vainio ${ }^{1,2,3}$ - the corresponding author; Email: lari.vainio@helsinki.fi; Tel: +358-2-94129392

Mikko Tiainen² (mikko.o.tiainen@helsinki.fi)

Kaisa Tiippana ${ }^{2}$ (kaisa.tiippana@helsinki.fi)

Martti Vainio ${ }^{3}$ (martti.vainio@helsinki.fi)

1 - Helsinki Collegium for Advanced Studies, University of Helsinki, Fabianinkatu 24, 00014, Finland.

2 - Perception, Action \& Cognition Research Group, Department of Psychology and Logopedics, Faculty of Medicine, University of Helsinki, Haartmaninkatu 8, 00014, Finland

3 - Phonetics and speech synthesis research group, Department of Digital Humanities, University of Helsinki, Unioninkatu 40, 00014, Finland 


\begin{abstract}
It has been shown recently that when participants are required to pronounce a vowel at the same time with the hand movement, the vocal and manual responses are facilitated when a front vowel is produced with forward-directed hand movements and a back vowel is produced with backwarddirected hand movements. This finding suggests a coupling between spatial programing of articulatory tongue movements and hand movements. The present study revealed that the same effect can be also observed in relation to directional leg movements. The study suggests that the effect operates within the common directional processes of movement planning including at least tongue, hand and leg movements, and these processes might contribute sound-to-meaning mappings to the semantic concepts of 'forward' and 'backward'.
\end{abstract}

Keywords: hand movements, leg movements, articulation, vowel production, reaction time 


\section{Introduction}

Production of articulatory gestures in speech might be the most complex motor programming task performed by humans. Perhaps the only other motor programming tasks that operate at the same level of complexity are related to controlling manual grasping and manipulation actions. Interestingly, there seems to be in fact a tight interaction between processes that program hand and mouth actions [1]. Correspondingly, the recent gestural theories of language evolution [2] assume that motor mechanisms that initially operated for manual gesturing and grasping might have contributed to the evolution of speech. In addition, most of the studies showing interaction between hand and mouth actions have focused on exploring how grasping in particular influences communicative $[3,4]$ and non-communicative [5] mouth movements. A variety of evidence shows that there are indeed systematic interactions between processes involved in planning precision and power grasps and certain articulatory gestures $[4,5]$.

Similarly to the effect showing the interaction between grasping and articulation, we have also recently shown that vowel production is connected to processes that prepare forwardbackward hand movements [6,7]. In that paradigm, the participants were presented with a front (e.g., [ø]) or back (e.g., [o]) vowel in the monitor. They were required to pronounce the vowel and simultaneously move the joystick either forward or backward according to the color in which the vowel was presented. The effect showed that manual and vocal responses were facilitated when the hand was moved in the same direction as the tongue in vowel fronting or backing. That is, the vowelreach effect shows that a front vowel is associated with forward hand movements while a back vowel is associated with backward hand movements.

It has to be also emphasized that this vowel-reach effect is observed even though participants are not explicitly required to move the tongue to any particular direction. Rather, this directional information appears to be implicitly included in planning tongue movements required for 
producing specific vowels: the participants were mostly not aware that production of the vowels that they had to pronounce were differentially associated with fronting and backing. In addition, although the effect was originally observed in Finnish speakers [6], it has been, so far, also replicated in Czech speakers [8]. The fact that the same effects can be observed in two separate languages that belong to differentiated language families (Finnish is a Finno-Ugric/Uralic language, and Czech is a Slavic/Indo-European language) suggests that people have an implicit and language-independent tendency to link production of front vowels to forward-directed hand movements and back vowels to backward-directed hand movements. Finally, the effect cannot be observed when articulation requires moving the tongue backward or forward in order to produce the dorsal consonant $[\mathrm{k}]$ performed by raising the tongue body toward the back of the velum or the coronal consonant $[\mathrm{t}]$ produced by fronting the tongue tip toward high-front position [8]. Hence, Vainio et al. [7] suggested that this effect is exclusively linked to fronting and backing the tongue body for vowel production rather than shaping the tongue in order to articulate the consonants such as $[\mathrm{t}]$ and $[\mathrm{k}]$. As such, the effect provides additional evidence for the view that there is an overlap between processes that plan hand actions and certain articulatory gestures. The finding suggests that the hand-mouth overlap does not only exist between processes responsible for planning hand shape for grasping and mouth shape for articulation $[3,4,5]$ but there is also an overlap between processes that plan direction coordinates for hand and tongue movements.

The vowel-reach effect is not, however, the first finding revealing that performance is improved when different body parts are moved in the same rather than opposite directions. It has been earlier shown that simultaneously performed symmetrical movements of upper limbs are executed more easily than asymmetrical movements. For example, it is easier to move both arms towards or away from the body midline than in different directions $[9,10]$. What makes the vowel-reach effect an exceptional phenomenon is that it shows that not only are movements of different limbs moved preferably in the same direction but directional hand and tongue 
movements, in vowel fronting and backing, also partially share an overlapping directional action planning processes. This finding is in line with the evidence showing that the same premotor regions appear to be involved in spatial programming of hand and tongue movements $[11,12]$.

\section{The present study}

The present study investigates whether this vowel-reach effect is exclusively related to processes responsible for directional planning of hand actions or whether the effect can be generalized to processes involved in planning forward-backward leg movements. Previous literature mostly emphasizes tight connection between motor processes of hand and mouth movements [13]. For instance, it has been reported that, in mice, tongue protrusions often co-occur with hand reaching movements [14], and that human infants make tongue protruding movements toward interesting objects such as pieces of food, presented within reaching distance, before they are old enough to successfully reach ( $<5$ months) [15]. Moreover, many accounts presume that these hand-mouth interactions are evolved to serve food intake [16]. As such, it is possible that the vowel-reach effect, as well as other similar hand-mouth effects [4] reflects evolutionarily old interactions in programming hand and tongue movements that originally evolved to serve a food intake function.

However, it has been also reported that during the pre-reaching stage, infants do not only aim to approach the interesting object by mouth movements, but this forward aiming also includes movements of legs [17]. In addition, Liuzzi, Ellger, Flöel, Breitenstein, Jansen and Knecht [18] have shown that corticospinal excitability of bilateral leg muscle representations are enhanced by speech production and silent reading. According to Liuzzi et al. [18], this finding demonstrates that the motor network involved in speech processes includes not just the mouth and hand motor representations but also the motor representations of other body parts, or at least 
motor representations of the legs. Furthermore, it has been also shown that when participants are required to produce directionally symmetric or asymmetric movements with two separate limbs, not only are symmetric hand movements performed more easily $[9,10]$, but also symmetric movements between hand and leg are performed more easily $[19,20]$. Hence, it appears that spatially oriented movement programming of a hand and leg share the same direction code at some level of action planning.

Taken together, previous research shows that directional movement planning processes of a hand and leg share the same directional codes $[19,20]$, and that the motor network involved in speech processes might include not just the mouth and hand motor representations [13] but also the motor representations of legs [18]. Consequently, it is plausible that the vowel-reach effect, which has previously revealed that the shared direction codes are utilized by directional processes that plan tongue and hand movements [6,7], could also be observed in relation to leg movements. Therefore, the study investigates whether the effect could be observed when the hand responses are replaced by leg responses. Hence, in the study, the participants were required to perform forward or backward directed hand or leg movements simultaneously with pronunciation of the front vowel [i] of the back vowel [o]. These vowels were selected to this study because all our participants were Finnish speaking individuals, and in Finnish the word 'forward' is 'eteen' ([ete:n]) while the word 'backward' is 'taakse' ([ta:kse]). That is, these two vowels are not included in these words, and hence any potential vowelreach effects that we might observe cannot be caused by links between the vocalized vowels and the letters that are used to write these two words. 


\section{Method}

\section{Participants}

Eighteen naïve volunteers participated in the study (20-32 years of age; mean age $=24.4$ years; 2 males; 1 left-handed). All participants were native speakers of Finnish and had normal or correctedto-normal vision and hearing. We obtained a written informed consent from all participants. The study was approved by the Ethical Review Board in Humanities and Social and Behavioural Sciences at the University of Helsinki.

\section{Apparatus, stimuli and procedure}

The main structure of the response device was built from two pieces of plywood panels. The smaller panel (vertically $32 \mathrm{~cm}$; horizontally $14 \mathrm{~cm}$ ) was attached to the larger panel (vertically $38 \mathrm{~cm}$; horizontally $14 \mathrm{~cm}$ ) with two metal rails so that the smaller panel moved over the larger one, which was attached to the floor (for leg responses) or table (for hand responses), smoothly and in a straight line. There were walls, constructed from a piece of plywood, attached at both ends of the lower panel (height $4 \mathrm{~cm}$ ) to provide a stopper for the movement of the upper panel. Pieces of soft foam plastic were glued onto both stopper walls. In addition, a knitting elastic band was attached between the upper panel and the stopper wall in both ends. These two construction elements pushed the upper panel to the vertically central position with respect to the lower panel. They also provided a minor resistance to the movement when the upper panel was moved toward the one or the other stopper wall. Micro-switches were also glued onto both stopper walls, and the end of the upper panel pushed the switch when the panel was moved forward or backward, until the end of the full motion range of the panel ( $3 \mathrm{~cm}$ to both directions). A piece of plywood (height $2 \mathrm{~cm}$ ) was attached on the upper panel, 6 
$\mathrm{cm}$ from the front end of it, in order to provide a grip for moving the panel. It was equally comfortable to grasp the grip and move the panel with the hand and foot.

Each participant sat in a dimly lit room with his or her head $65 \mathrm{~cm}$ from a 19 -in. CRT monitor (screen refresh rate: $85 \mathrm{~Hz}$; screen resolution: $1280 \times 1024)$. The head-mounted microphone was adjusted close to the participant's mouth for recording vocal responses. The study consisted of two blocks that were separated by a short break. In one block the participants responded by moving the upper panel of the response device with their right hand and in the other they responded by moving the upper panel with their right leg. In the hand block, the response device was attached on the table with Blu-Tack in front of the monitor. When the panel was moved forward/backward with the arm, the movement was predominantly executed with the elbow extension/flexion, respectively. In the leg block, the device was attached onto the floor with Blu-Tack. The participants were seated so that their shins and thighs formed $90^{\circ}$ angles when the upper panel was at the vertically neutral position. These arrangements ensured that when the panel was moved forward/backward with the foot, the movement was predominantly executed with the knee extension/flexion, respectively.

Both blocks consisted of two different target vowels [i] and [o] that were presented at the center of the monitor. Half of the vowels were presented in green color and the other half were presented in blue color. The vowels were written in Consolas font (lowercase; bold; font size: 72). All stimuli were displayed on a gray background. The vowels were presented in randomized order within both blocks. In addition, the order of blocks was balanced between the participants. The response directions were marked with corresponding color tapes on the response device. Half of the participants responded to the green by moving the panel forward and to the blue by moving it backward, and other half responded vice versa. The panel was always returned to the central starting position after the response. Erroneous manual and leg responses were immediately followed by a short 'beep' tone. In total, the experiment consisted of 240 trials [30 x 2 (vowel) x 2 (response) x 2 (block)]. 
At the beginning of each trial, a fixation cross was presented for $400 \mathrm{~ms}$ in the center of the screen. Then, a blank screen was displayed for $400 \mathrm{~ms}$. After that the target vowel was presented in the center of the screen and remained in view for $1000 \mathrm{~ms}$ or until a response was made. Finally, a blank screen was displayed for $500 \mathrm{~ms}$. The participants were instructed to pronounce the presented vowel as fast as possible. It was emphasized that the vowel should be uttered in natural talking voice at the same time with the hand/leg response. The actual experiment was not started before the participant demonstrated in the practice session that he/she consistently produced the vocal and hand/leg responses at the same time. The recording levels of the vocal responses were calibrated individually for each participant at the beginning of the experiment. Stimulus presentation and sound recording were done with Presentation 16.1 software.

Manual reaction times were measured from the onset of the target stimulus until the moved panel pushed the micro-switch. The vocal responses were recorded for $2000 \mathrm{~ms}$ starting from the onset of the target object. Vocal data were analyzed using Praat v. 5.3.49 (http:// www.praat.org). Onsets of vocalization were located individually for each trial as the first observable peak in the acoustic signal for the vowel.

\section{Results and discussion}

In all analysis described below, condition means for the data that remained after removing trials containing errors and in which the RTs were more than two standard deviations from a participant's overall mean (see [21] for a justification of using this data-trimming method) were subjected to a repeated-measures ANOVA with the within-participants factors of Vowel ([i], [o]) and Movement direction (MD: forward, backward). Post hoc comparisons were performed by means of t-tests applying a Bonferroni correction when appropriate. A partial-eta-squared statistic served as an effect size estimate. 


\section{Block 1: Hand movements}

Movement reaction times: In total, $5 \%$ of the raw data were discarded from the RT analysis including $0.5 \%$ of trials containing manual errors, $0.1 \%$ of trials containing vocal errors, $0.2 \%$ of trials containing no vocal or manual responses and $4.2 \%$ of trials in which the RTs were more than two standard deviations from a participant's overall mean.

The analysis of movement reaction times revealed a significant interaction between Vowel and MD, $\mathrm{F}(1,17)=12.99, \mathrm{MSE}=14863.50, \mathrm{p}=.002, \eta_{\mathrm{p}}{ }^{2}=.433$. The forward responses were made faster when the vowel was [i] $(\mathrm{M}=595 \mathrm{~ms})$ rather than $[\mathrm{o}](\mathrm{M}=621 \mathrm{~ms})(\mathrm{p}=.002)$, whereas backward responses were made faster when the vowel was [o] $(\mathrm{M}=579 \mathrm{~ms})$ rather than $[\mathrm{i}](\mathrm{M}=610$ $\mathrm{ms})(\mathrm{p}=.012)$. The interaction is presented in Fig 1 . The error data were not analyzed due to low error rates.

Vocal reaction times: These data were analyzed in the same way as the movement reaction times. In total, $5.1 \%$ of the raw data was discarded from the RT analysis including $0.5 \%$ of trials containing manual errors, $0.1 \%$ of trials containing vocal errors, $0.2 \%$ of trials containing no vocal or manual responses and $4.3 \%$ of trials in which the RTs were more than two standard deviations from a participant's overall mean.

The analysis of vocal reaction times revealed a significant interaction between Vowel and $\mathrm{MD}, \mathrm{F}(1,17)=30.39, \mathrm{MSE}=18133.71, \mathrm{p}<.001, \eta_{\mathrm{p}}{ }^{2}=.641$. The vowel $[\mathrm{i}]$ was pronounced faster with forward movements $(M=603 \mathrm{~ms})$ than with backward movements $(M=635 \mathrm{~ms})(\mathrm{p}<.001)$. In contrast, the vowel [o] was pronounced faster with backward movements $(\mathrm{M}=599 \mathrm{~ms})$ than with forward movements $(\mathrm{M}=631 \mathrm{~ms})(\mathrm{p}=.002)$. The interaction is presented in Fig 1 . The error data was not analyzed due to small error rates. 


\section{Block 2: Leg movements}

Movement reaction times: In total, $8 \%$ of the raw data were discarded from the RT analysis including $2.6 \%$ of trials containing leg errors, $0.2 \%$ of trials containing vocal errors, $0.5 \%$ of trials containing no vocal or leg responses and $4.7 \%$ of trials in which the RTs were more than two standard deviations from a participant's overall mean.

The analysis of movement reaction times revealed a significant interaction between Vowel and $\mathrm{MD}, \mathrm{F}(1,17)=19.59, \mathrm{MSE}=27208.38, \mathrm{p}<.001, \eta_{\mathrm{p}}{ }^{2}=.535$. The forward responses were made faster when the vowel was [i] $(\mathrm{M}=563 \mathrm{~ms})$ rather than $[\mathrm{o}](\mathrm{M}=602 \mathrm{~ms})(\mathrm{p}=.009)$, whereas backward responses were made faster when the vowel was $[\mathrm{o}](\mathrm{M}=580 \mathrm{~ms})$ rather than $[\mathrm{i}](\mathrm{M}=618$ $\mathrm{ms})(\mathrm{p}=.001)$. The interaction is presented in Fig 1 . The error data was not analyzed due to small error rates.

Vocal reaction times: These data were analyzed in the same way as the movement reaction times. In total, $7.7 \%$ of the raw data was discarded from the RT analysis including $2.6 \%$ of trials containing manual errors, $0.2 \%$ of trials containing vocal errors, $0.5 \%$ of trials containing no vocal or manual responses and $4.4 \%$ of trials in which the RTs were more than two standard deviations from a participant's overall mean.

The vocal reaction times revealed a significant interaction between Vowel and MD, $\mathrm{F}(1,17)=19.33, \mathrm{MSE}=16803.30, \mathrm{p}<, .001, \eta_{\mathrm{p}}{ }^{2}=.532$. The vowel [i] was pronounced faster with forward movements $(\mathrm{M}=584 \mathrm{~ms})$ than with backward movements $(\mathrm{M}=617 \mathrm{~ms})(\mathrm{p}=.004)$. In contrast, the vowel [o] was pronounced faster with backward movements [i] $(\mathrm{M}=591 \mathrm{~ms})$ than with forward movements $(\mathrm{M}=618 \mathrm{~ms})(\mathrm{p}=.009)$. The interaction is presented in Fig 1 . The error data were not analyzed due to low error rates.

---Figure 1 about here--- 


\section{Discussion}

The results of the study showed that the vowel-reach effect, previously observed between forward/backward hand movements and front/back vowels [6,7], is also observed when the hand responses are replaced by forward/backward leg movements. In other words, production of a front vowel is preferably coupled with upper and lower limb movements that are also directed forwards whereas production of a back vowel is preferably coupled with upper and lower limb movements that are directed backwards. Hence, the effect appears to operate within common and abstract directional motor programming processes rather than within specific connections between hand and mouth motor processes. In that sense, the effect is similar to those phenomena showing preference for performing directionally symmetric upper and lower limb movements [19,20]. However, as an addition to the findings showing common directional planning of movements of different limbs, the present study suggests that directional components of tongue motor programs related to vowel production are, to some extent, prepared in interaction with the same abstract directional coding that serves limb movements.

Chieffi, Secchi, \& Gentilucci [22] have previously shown that forward hand movements are automatically associated with an Italian word that semantically points away from the participants' body ('LA' = 'there') while backward hand movements are associated with a word that points toward the participants' body ('QUA' = 'here'). However, the present study shows that mechanisms responsible for pronunciation can influence forward-backward hand movements even when the semantic information is removed. In this case, the effect is based on shared non-semantic directional codes utilized for producing articulatory gestures and limb movements. An interesting question, however, is whether this involvement of common directional coding in vowel production might nevertheless manifest itself in semantic and lexical context. We propose that the network representing common directional codes is not utilized just for orienting body parts such as hand, leg and tongue toward the same direction but might be also a central component in a conceptual representation of 
direction. This kind of tendency to link vowel frontness/backness to direction-related concepts might also manifest itself in a universal tendency to include front vowels in the words that are semantically forward-directed or in the deictic words that are semantically pointing away from an individual's own body. In contrast, back vowels might be associated with the words that are semantically backwarddirected or the deictic words that are semantically pointing toward an individual's own body. In turn, these tendencies might have gradually modulated lexicon at the universal scale. In fact, this view is supported by a cross-linguistic study that has indeed revealed that the back vowel $[a]$ is relatively frequently included in the pronoun that points to the speaker ('I') whereas the front vowel [i] is more frequently included in the pronoun that points to the hearer ('you') [23].

It has been recently demonstrated that back vowels are implicitly associated with the concept of 'far' while front vowels are associated with the concept of 'near' [24]. This finding was proposed to be linked to the way these vowels are articulated. That is, the back vowels are produced by lengthening the oral cavity, which in turn provides "iconic" oral gesture for lengthened distance. Similarly, the front vowels are produced by shortening the oral cavity, which in turn provides a gestural analogy for short distance. Given that the vowel-reach effect can be observed in relation to upper as well as lower limb movements, the vowel-reach effect might provide other example of this kind of gesture-to-meaning mapping in which specific articulatory movement in relation to vowel production is implicitly mapped to a specific semantic concept. In the current case, the forwarddirected articulatory tongue movement is mapped to the concept of 'forward' while the backwarddirected articulatory tongue movement is mapped to the concept of 'backward'.

One way to look at the present results is in terms of a common reference frame for movement plans of multiple effectors [25]. This account assumes that even though the posterior parietal cortex (PPC) contains functional regions that are specialized for planning movements of different effectors (e.g., eyes, head and hand), the spatial goal of action can be represented in the PPC within a common reference frame, which facilitates coordination of the movements of different 
effectors in the same direction. Hence, if one interprets the present results in terms of this account, it could be assumed that these PPC processes also facilitate planning movements of tongue, head and limbs in the same direction. As such, it would be interesting to test whether the same effect were observed if the current task, which requires moving upper and lower limbs while producing different vowels, were replaced by the task in which forward-backward head movements were performed instead of limb movements.

In conclusion, the present study showed that the vowel-reach effect operates within the common directional planning processes that couple planning of directional vowel-related tongue movements with directional limb movements. That is, direction components for articulatory tongue movements are not programmed in isolation but share processes, at least, with planning of hand and leg movements. In addition, the present findings suggest that the processes related to planning the direction of goal directed actions in a combined form for articulatory tongue movements and limb movements might contribute to semantic representation of directional concepts such as "forward" and "backward".

\section{Acknowledgement and funding details}

The research leading to these results has received funding from the Academy of Finland under grant agreement numbers 1265610 . We would like to thank Aleksi Rantala for his contribution in collecting the data. 


\section{References}

[1] Iverson, J. M., \& Thelen, E. (1999). Hand, mouth and brain. The dynamic emergence of speech and gesture. Journal of Consciousness Studies, 6, 19-40.

[2] Rizzolatti, G., \& Arbib, M.A. (1998). Language within our grasp. Trends in Neuroscience, 21, 188-194.

[3] Gentilucci, M., \& Campione, G.C. (2011). Do Postures of Distal Effectors Affect the Control of Actions of Other Distal Effectors? Evidence for a System of Interactions between Hand and Mouth. PLoS ONE, 6, 5: e19793.

[4] Vainio, L., Schulman, M., Tiippana, K., \& Vainio, M. (2013). Effect of syllable articulation on precision and power grip performance. PLoS ONE 8(1), e53061.

[5] Gentilucci, M., Benuzzi, F., Gangitano, M. \& Grimaldi, S. (2001). Grasping with hand and mouth: a kinematic study on healthy subjects. Journal of Neurophysiology, 86, 1885-1699.

[6] Vainio, L., Tiainen, M., Tiippana, K., Komeilipoor, N., \& Vainio, M. (2015). Interaction in planning movement direction for articulatory gestures and manual actions. Experimental Brain Research, 233, 2951-2959.

[7] Vainio, L., Tiippana, K., Tiainen, M., Rantala, A., \& Vainio, M. (2018). Reaching and grasping with the tongue: Shared motor planning between hand actions and articulatory gestures. Quarterly Journal of Experimental Psychology, 1747021817738732.

[8] Tiainen, M., Lukavský, J., Tiippana, K., Vainio, M., Šimko, J., Felisberti, F., \& Vainio, L. (2017). Systematic connections of grasping and horizontal hand movements with articulation in Czech speakers. Frontiers in Psychology, 8, 516.

[9] Wiesendanger, M., Wicki, U., Rouiller, E. (1994). Are there unifyingstructures in the brain responsible for interlimb coordination? in S.P. Swinnen, H. Heuer, J. Massion, P. Casaer (Eds.), Interlimb Coordination: Neural, Dynamical, and Cognitive Constraints, Academic Press, San Diego, pp. $179-207$. 
[10] Lee, T.D., Swinnen, S.P., \& Verschueren, S. (1995). Relative phase alterations during bimanual skill acquisition. Journal of Motor Behavior, 27, 263 - 274.

[11] Kawashima, R., Itoh, H., Ono, S., Satoh, K., Furumoto, S., Gotoh, R., ... \& Yanagisawa, T. (1996). Changes in regional cerebral blood flow during self-paced arm and finger movements. A PET study. Brain Research, 716(1-2), 141-148.

[12] Watanabe, J., Sugiura, M., Miura, N., Watanabe, Y., Maeda, Y., Matsue, Y., \& Kawashima, R. (2004). The human parietal cortex is involved in spatial processing of tongue movement—an fMRI study. Neuroimage, 21(4), 1289-1299.

[13] Meister, I. G., Boroojerdi, B., Foltys, H., Sparing, R., Huber, W., \& Töpper, R. (2003). Motor cortex hand area and speech: implications for the development of language. Neuropsychologia, 41, 401-406.

[14] Shisha, I. Q., Agha, B. M., Kuntz, J. R., Faraji, J., \& Mohajerani, M. H. (2018). Tongue protrusions modify the syntax of skilled reaching for food by the mouse: Evidence for flexibility in action selection and shared hand/mouth central modulation of action. Behavioural Brain Research, $341,37-44$.

[15] Jones, S. S. (1996). Imitation or exploration? Young infants' matching of adults' oral gestures. Child development, 67, 1952-1969.

[16] Gentilucci, M., \& Corballis, M. C. (2006). From manual gesture to speech: A gradual transition. Neuroscience \& Biobehavioral Reviews, 30, 949-960.

[17] Foroud, A., \& Whishaw, I. Q. (2012). The consummatory origins of visually guided reaching in human infants: a dynamic integration of whole-body and upper-limb movements. Behavioural Brain Research, 231, 343-355.

[18] Liuzzi, G., Ellger, T., Flöel, A., Breitenstein, C., Jansen, A., \& Knecht, S. (2008). Walking the talk-Speech activates the leg motor cortex. Neuropsychologia, 46, 2824-2830. 
[19] Baldissera, F., Cavallari, P., \& Civaschi, P. (1982). Preferential coupling between voluntary movements of ipsilateral limbs. Neuroscience Letters, 34, 95-100.

[20] Kelso, J. A., \& Jeka, J. J. (1992). Symmetry breaking dynamics of human multilimb coordination. Journal of Experimental Psychology: Human Perception and Performance, 18, 645.

[21] Singh, G. (2006). Determination of cutoff score for a diagnostic test. The Internet Journal of Laboratory Medicine, 2, 1-4.

[22] Chieffi, S., Secchi, C., \& Gentilucci, M. (2009). Deictic word and gesture production: Their interaction. Behavioural Brain Research, 203, 200-206.

[23] Wichmann, S., Holman, E. W., \& Brown, C. H. (2010). Sound symbolism in basic vocabulary. Entropy, 12, 844-858.

[24] Rabaglia, C. D., Maglio, S. J., Krehm, M., Seok, J. H., \& Trope, Y. (2016). The sound of distance. Cognition, 152, 141-149.

[25] Cohen, Y. E., \& Andersen, R. A. (2002). A common reference frame for movement plans in the posterior parietal cortex. Nature Reviews Neuroscience, 3, 553-562. 
Figure

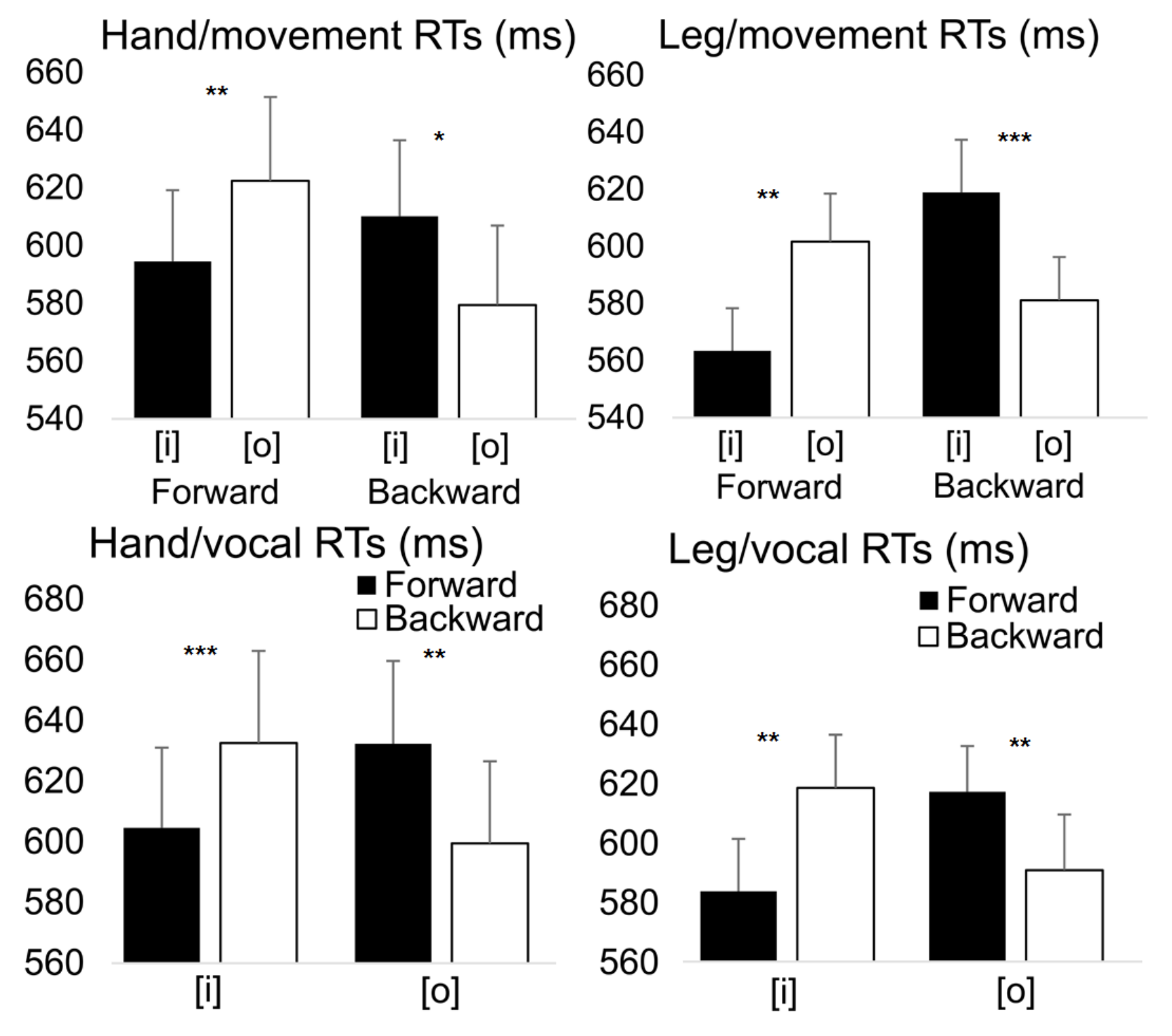

Figure 1. The mean manual and vocal reaction times for the study as a function of the vowel and the movement direction. The histograms on the left are showing the mean reaction times when the responses were performed with the hand, while the histograms on the right are showing the mean reaction times when the responses were performed with the leg. Error bars depict the standard error of the mean. Asterisks indicate statistically significant differences $\left(* * *<.001 ; * * p<.01 ;{ }^{*} p<.05\right)$. 